\title{
WHITE LIGHT PHOTOMETRY OF THE CATACLYSMIC VARIABLE BZ CAMELOPARDALIS
}

\author{
Gabriel PAJDOSZ ${ }^{\dagger}$ and Stanisław ZOLA ${ }^{\ddagger}+$ \\ ${ }^{\dagger}$ Mt. Suhora Observatory, Pedagogical University \\ u1. Podchorazych 2, 30-084 Cracow, Poland \\ *Astronomical Observatory, Jagiellonian University \\ ul. Orla 171, 30-244 Cracow, Poland
}

\begin{abstract}
We present high-speed photoelectric observations of a 13th-mag cataclysmic binary BZ Cam $(=0623+71)$, gathered in 1989 and 1990. The data were analyzed by means of the Fourier technique. The most prominent feature detected in the power spectrum is a $3^{\mathrm{h}} 36^{\mathrm{m}}$ period which we ascribe as being a photometric orbital modulation. This period is significantly different from the periodicity previously known from spectroscopic observations. The power spectrum shows also quasi-periodic oscillations of shorter time-scales but any conclusions about their stability can not be drawn yet.
\end{abstract}

\section{Introduction}

The BZ Cam system (positional designation 0623+71), due to its complex nature, has been an object of several studies since its discovery reported by Ellis et al. (1984). It shows properties of a cataclysmic variable surrounded by a faint emission nebula. From spectra published by Williams (1983), Garnavich and Szkody (1988) deduced some evidence for existence of a disk in BZ Cam. Radial velocity observations carried out by Lu and Hutchings (1985) supported the binary nature of BZ Cam and led to two most probable orbital periods of the variable: 0.1390 and 0 .1535. Direct CCD images and long-slit spectrograms of the whole system have been presented by Krautter et al. (1987). The authors more or less discarded the possibility of BZ Cam being either a central star of a planetary nebula or an old-nova object. Woods et al. (1990) found from UV spectroscopic studies the effects of a high-velocity wind in the system. The photographic long-time behaviour of the system's brightness completed by Garnavich and Szkody (1988) showed the presence of low-states in BZ Cam and they classified it as a member of VY Scl group of cataclysmic variables. They derived the mean high-state $B$ magnitude to be 12.7 and the high- to low-state amplitude about 1.5 .

\section{Observations}

The observations were carried out with a $0.6 \mathrm{~m}$ Cassegrain telescope at Mt. Suhora Observatory of the Cracow Pedagogical University, which was 
equipped with a double beam photometer. A thorough discussion of the instrument configuration and examples of its operation has been published by Szymanski and Udalski (1989). The photoelectric data of BZ Cam were obtained during October 27 and 28, 1989 and October 10, 11 and 12 , 1990. The duration of individual runs varies from 6.9 to 9.8 hours. The data were gathered in white light (extended S20 photocatode response) using $30 \mathrm{~s}$ integrations. $\mathrm{BD}+71^{\circ} 352$ ( $\mathrm{Sp} . \mathrm{A} 5, \mathrm{~m}=10^{\mathrm{m}} .0$ ) has been chosen as the comparison star. The observations were reduced in the standard way, they were left uncalibrated and were corrected for differential extinction using mean extinction coefficient for B filter. An error of an individual observation of the variable star did not exceed 0.01 .

The optical light curves of BZ Cam are shown in Figs $1 \mathrm{a}$ and $1 \mathrm{~b}$. There
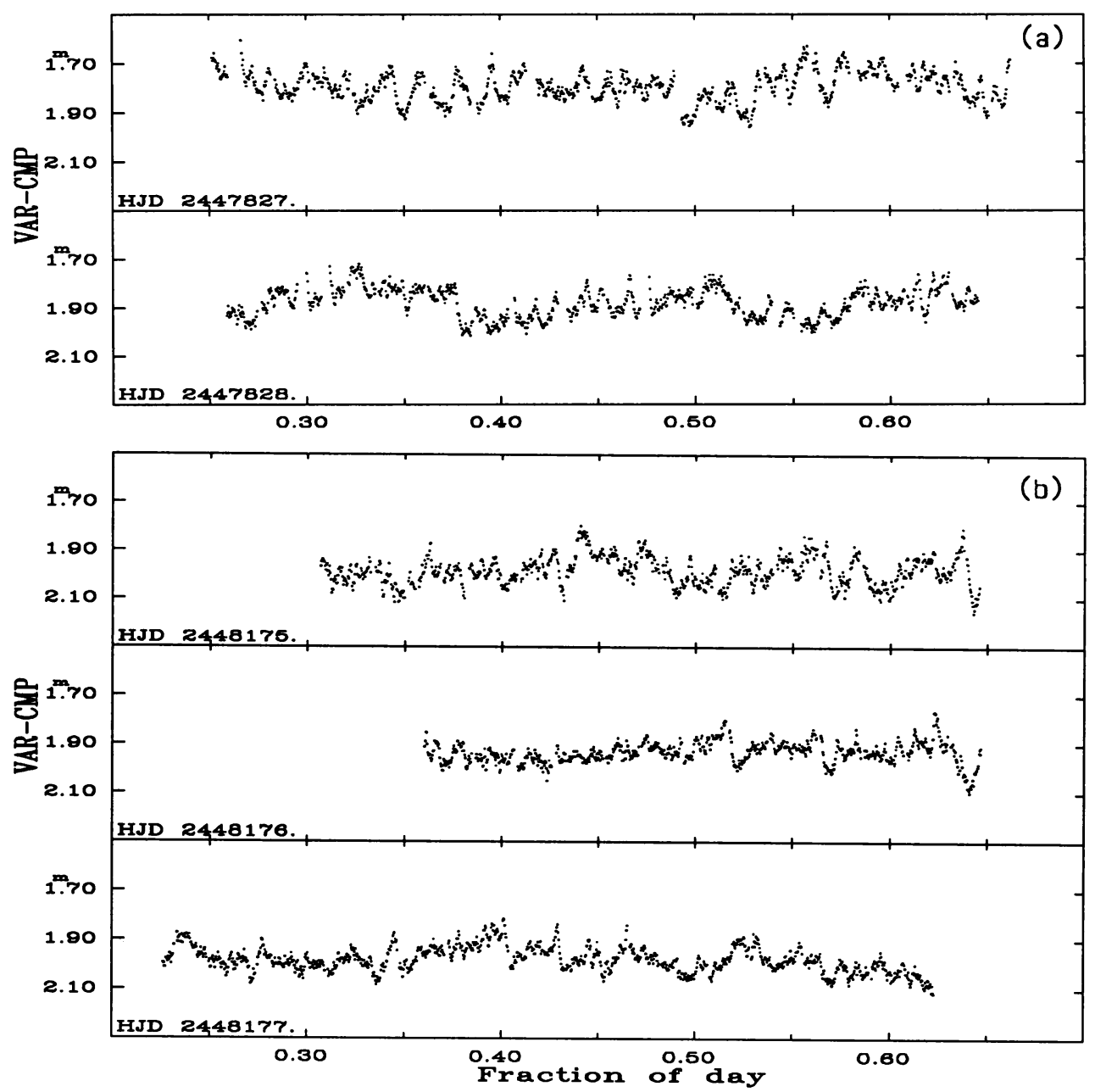

Figure 1a and b. The white-light curves of BZ Cam obtained on 27th and 28th October 1989 (a) and on 10th, 11th and 12th October 1990 (b). 
is no evidence of eclipses and a periodic modulation of order of hours is evident in all runs presented. One can notice that photoelectric behaviour of the star is quite different during the two seasons. During 1989 season the light curve shows clear and large amplitude variations on time scale of 20-30 min. Other striking features are sudden drops of light visible in the presented data. During 1990 season the amplitude of variations is smaller and the dominant is only a modulation of order of hours. Short-time behaviour seems to be more complicated and probably fully contaminated by the random flickering. The comparable difference between variable and comparison stars (see Figs 1a and 1b) suggests that the system was observed in the same state.

\section{Fourier Analysis}

The data have been analyzed using Kurtz' (1985) modified algorithm of the Deeming (1975) Discrete Fourier Transform technique. For each night an average of all observations was calculated and subtracted from individual data to remove the effects of variability on time scales $>1$ day. Next, the Fourier power spectra were computed for separate runs as well as for all nights of the season together, in the frequency range from 0 to $300 \mathrm{c} / \mathrm{d}$. The seasonal power spectra are presented in Figs $2 \mathrm{a}$ and $2 \mathrm{~b}$. Additionally, we computed power spectra for uncorrected comparison and variable star observations to check if there is any evidence of intrinsic variability of the former star. Apart from the atmospherical effects we found no such variability.

The most prominent feature we detected is the 3.6 hour $\left(f_{0}=6.7 \mathrm{c} / \mathrm{d}\right)$ modulation as well as frequencies at its 1 st and $3 \mathrm{rd}$ harmonics $\left(2 \mathrm{f}_{0}=13.4\right.$ $\mathrm{c} / \mathrm{d}$ and $4 \mathrm{fo}_{\mathrm{o}}=26.8 \mathrm{c} / \mathrm{d}$ respectively). The peaks corresponding to these periods appear in all individual night's power spectra. The appearance of harmonics indicate the nonsinusoidal shape of the main modulation.

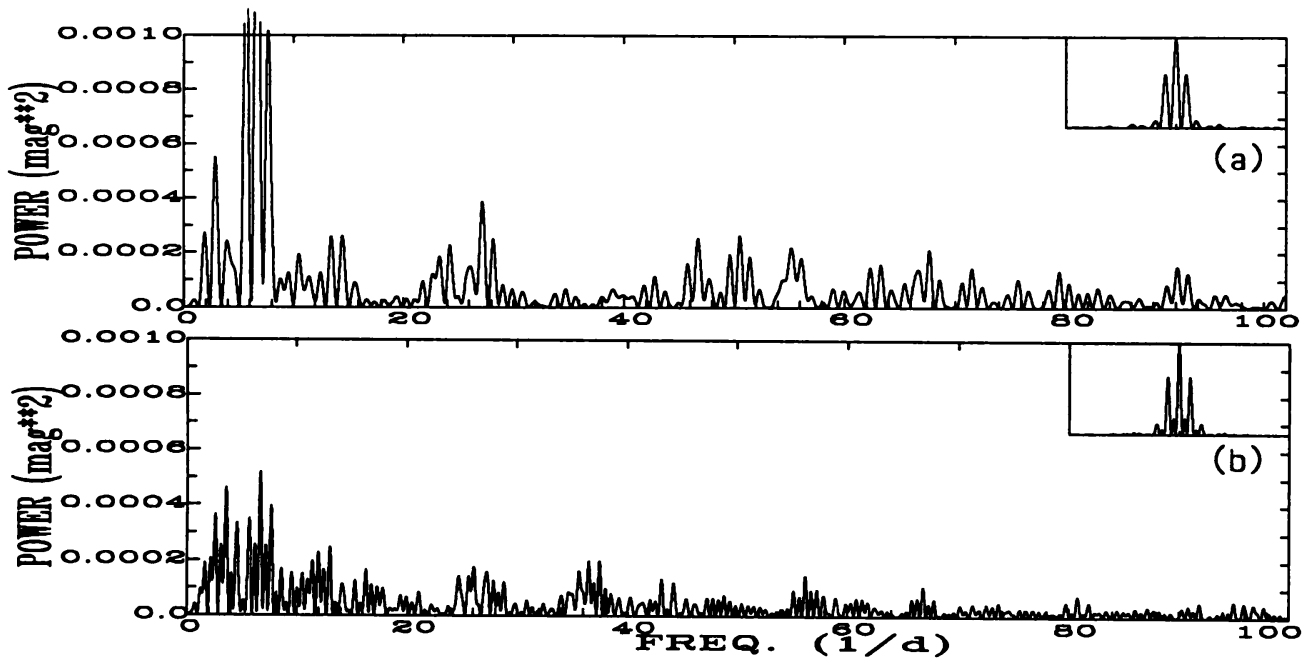

Figure $2 a$ and $b$. Fourier power spectra of BZ Cam for observations obtained in 1989 (a) and 1990 (b). Inset: the spectral windows. 
The least squares sine-curve fitted to the data provides the following ephemerides of the 3.6 hour period for 1989 and 1990 seasons:

$$
\begin{aligned}
& \text { 1989: HJD Max }=2447827.581(14)+0.15006(25) \mathrm{E} \\
& \text { 1990: HJD Max }=2448175.436(10)+0.15011(25) E
\end{aligned}
$$

The obtained fits yield also a white-light amplitude of this modulation being $0^{\mathrm{m}} .045(2)$ in 1989 and $0^{\mathrm{m}} .023(2)$ in 1990.

Because we found no discrepancies between these two above periods we fitted the sine-curve to all data available. The most likely period we found is equal to: $0.1500023(7)$. We must point out here the discrepancy between the spectroscopic estimations of the orbital period and our results. We tried to phase the spectroscopic observations published by Lu and Hutchings (1985) with our period but the scatter of such phased data was much larger than those phased with the original periods. Thus, we believe the discrepancy is real. This phenomenon is typical of intermediate polars and SU UMa groups of cataclysmic variables (see e.g. Udalski 1988, Udalski and Schwarzenberg-Czerny 1989).

We searched also for other periodicities in the data. The $\sim 3 \mathrm{c} / \mathrm{d}$ peak corresponds to the length of data and arises from atmospheric effects and a crude reduction procedure used for taking into account atmospheric extinction. A quasi-periodic oscillations are detected on time-scales less than an hour $(\sim 40-80 \mathrm{c} / \mathrm{d})$ but they are not present in all the nights analyzed or/and they are not stable in the frequency domain. Because of insufficient observational data we are not able to draw any general conclusions about the short-term variability in BZ Cam yet.

\section{Conclusions}

The difference between the orbital and photometric periods, the existence of disc around the white dwarf, the length of the period (longer than 2.5 corresponding to the period gap) and the long term behaviour suggest that BZ Cam could be classified as an intermediate polar. Since the rotation frequency of the white dwarf is unknown, as well as there is no additional information available from $X$-ray and polarization measurements, the classification character of the system is still questionable.

\section{Acknowledgements}

We are grateful to $\mathrm{Dr}$ A.Schwarzenberg-Czerny for suggesting the subject. We would like to thank to Prof. J.Smak for stimulating discussion.

\section{REFERENCES:}

Deeming T.J.: 1975, Astrophys.Space Sci. 36, 137.

Ellis G.L., Grayson E.T., Bond H.E.: 1984, Publ.Astr.Soc.Pacific 96, 283.

Garnavich P., Szkody P.: 1988, Publ.Astr.Soc.Pacific 100, 1522.

Krautter J., Klaas U., Radons G.: 1987, Astr. Astrophys. 181, 373.

Kurtz D.W.: 1985, Mon.Not.R. Astr.Soc. 213, 773.

Lu W., Hutchings J.B.: 1985, Publ.Astr.Soc.Pacific 97, 990.

Szymanski M., Udalski A.: 1989, Acta Astr. 39, 1.

Udalski A.: 1988, Acta Astr. 38, 315.

Udalski A., Schwarzenberg-Czerny A.: 1989, Acta Astr. 39, 125.

Williams G.: 1983, Astroph.J.Suppl., 53, 523.

Woods J.A., Drew J.E., Verbunt F.: 1990, Mon.Not.R. Astr.Soc. 245, 323. 